\title{
Ozsváth-Szabó and Rasmussen invariants of cable knots
}

\author{
CORnelia A VAn COTT
}

\begin{abstract}
We study the behavior of the Ozsváth-Szabó and Rasmussen knot concordance invariants $\tau$ and $s$ on $K_{m, n}$, the $(m, n)$-cable of a knot $K$ where $m$ and $n$ are relatively prime. We show that for every knot $K$ and for any fixed positive integer $m$, both of the invariants evaluated on $K_{m, n}$ differ from their value on the torus knot $T_{m, n}$ by fixed constants for all but finitely many $n>0$. Combining this result together with Hedden's extensive work on the behavior of $\tau$ on $(m, m r+1)$-cables yields bounds on the value of $\tau$ on any $(m, n)$-cable of $K$. In addition, several of Hedden's obstructions for cables bounding complex curves are extended.
\end{abstract}

$57 \mathrm{M} 25$

\section{Introduction}

The $(m, n)$-cable of a knot $K$, denoted $K_{m, n}$, is the satellite knot with companion $K$ and pattern $T_{m, n}$, the $(m, n)$-torus knot. The behavior of many classical concordance invariants has been shown to be rather predictable on cable knots. For example, it is a classical result (see Lickorish [6]) that the Alexander polynomial of a cable knot is

$$
\Delta_{K_{m, n}}(t)=\Delta_{K}\left(t^{m}\right) \Delta_{T_{m, n}}(t) .
$$

Shinohara [17] found a formula for the signature of a cable knot, and Litherland [7] extended the result, finding the value of Tristam-Levine signatures on a cable knot:

$$
\sigma_{\omega}\left(K_{m, n}\right)=\sigma_{\omega^{m}}(K)+\sigma_{\omega}\left(T_{m, n}\right) .
$$

Milnor signatures and Casson-Gordon invariants of cables (see Litherland [8] and Kearton [5], respectively, for details) also yield nice formulas.

The purpose of this note is to investigate two relatively new concordance invariants - the Ozsváth-Szabó invariant $\tau$ and the Rasmussen invariant $s$ - and their behavior on cable knots. The discussion here will use only the formal properties that the two invariants have in common.

Both $\tau$ and $s$ were introduced in connection with developments in the theory of knot homologies: $\tau$ is defined in terms of knot Floer homology (see Ozsváth and Szabó [10] and Rasmussen [15]) and the Rasmussen invariant $s$ is defined in terms of Khovanov homology (see Rasmussen [14]). These two invariants have enabled important progress 
in the field of knot theory, providing new proofs for Milnor's conjecture [10; 14] and examples of Alexander polynomial one knots which are not smoothly slice (see Livingston [9]).

No work has been done to compute the Rasmussen invariant for cables, but the behavior of the Ozsváth-Szabó concordance invariant $\tau$ under $(m, m r+1)$-cabling has been investigated by Hedden $[2 ; 3]$. Through careful investigation of the relationship between the filtered chain homotopy types of $\mathcal{F}\left(K_{m, m r+1}, i\right)$ and $\mathcal{F}(K, i)$, he obtained the following main result:

Theorem 1 [3] Let $K \subset S^{3}$ be a nontrivial knot. Then the following inequality holds for all $r$ :

$$
m \tau(K)+\frac{(m r)(m-1)}{2} \leq \tau\left(K_{m, m r+1}\right) \leq m \tau(K)+\frac{(m r)(m-1)}{2}+m-1 .
$$

In the special case when $K$ satisfies $\tau(K)=g(K)$, we have the equality

$$
\tau\left(K_{m, m r+1}\right)=m \tau(K)+\frac{(m r)(m-1)}{2},
$$

whereas when $\tau(K)=-g(K)$, we have

$$
\tau\left(K_{m, m r+1}\right)=m \tau(K)+\frac{(m r)(m-1)}{2}+m-1 .
$$

When appropriately normalized, $\tau$ and $s$ share several formal properties and agree on many families of knots, though in general they have been shown to be distinct invariants (see Hedden and Ording [4]). Stated in reference to $\tau$, the essential formal properties are as follows (see Ozsváth and Szabó [10]):

(1) $\tau$ is a homomorphism from the smooth knot concordance group $\mathcal{C}$ to $\mathbb{Z}$.

(2) $|\tau(K)| \leq g_{4}(K)$, where $g_{4}(K)$ denotes the 4-genus of $K$.

(3) $\tau\left(T_{m, n}\right)=(m-1)(n-1) / 2$, where $T_{m, n}$ denotes the $(m, n)$-torus knot with $m, n \geq 1$.

It can be shown that $s / 2$ also satisfies these three properties [14]. In addition, both $\tau$ and $s$ are insensitive to a change in orientation $[11 ; 14]$. Our main results will only depend on these formal properties, and hence apply to both invariants. To proceed concisely, let $v$ denote any concordance invariant satisfying the above properties.

Fixing $m>0$, we would like to study the value of $v$ on $K_{m, n}$ as a function of $n$, where $n$ ranges over the integers relatively prime to $m$. (Notice that $K_{m, n}=-K_{-m,-n}$, and so the restriction $m>0$ does not limit our results.) From our observations about other concordance invariants, we expect that the behavior of $v\left(K_{m, n}\right)$ as a function of $n$ is somehow related to the behavior of $v\left(T_{m, n}\right)$. This, in fact, is true. As a function of $n$, 
$v\left(T_{m, n}\right)$ is linear of slope $(m-1) / 2$ for $n>0$. We will see that the function $v\left(K_{m, n}\right)$ is close to being linear with the same slope. Specifically, we subtract from $v$ a linear function to construct the following function:

$$
h(n)=v\left(K_{m, n}\right)-\frac{(m-1)}{2} n,
$$

where $n$ is an integer relatively prime to $m$. We have the following theorem:

Theorem 2 The function $h(n)$ is a nonincreasing $\frac{1}{2} \cdot \mathbb{Z}$-valued function which is bounded below. In particular, we have

$$
-(m-1) \leq h(n)-h(r) \leq 0
$$

for all $n>r$, where both $n$ and $r$ are relatively prime to $m$.

From this result it follows that for all $n$ large enough, $h$ is constant. Hence for $n$ large enough, $v\left(K_{m, n}\right)$ differs from $v\left(T_{m, n}\right)$ by a fixed constant. That is, for every knot $K$ there exist integers $N$ and $c$ such that $v\left(K_{m, n}\right)=v\left(T_{m, n}\right)+c$ for all $n>N$, where $n$ is relatively prime to $m$. Additionally, a similar statement with corresponding constant $c^{\prime}$ holds for all $n<N^{\prime}$ for some $N^{\prime}$.

Theorem 2 is sharp in the sense that there are knots $K$ with associated functions $h$ which achieve the bounds given in the theorem. For example, when $K$ is slice, $h(n)=(m-1) / 2$ for all $n<0$ and $h(n)=-(m-1) / 2$ for all $n>0$. Here the drop in functional value from $n=-1$ to $n=1$ is maximal: $h(1)-h(-1)=-(m-1)$. On the other hand, we will see that when $v=\tau$ and $\tau(K)=g_{3}(K)$, the function $h$ is constant.

Using Theorem 2, we can take several results which apply only to $(m, m r+1)$-cables and extend their scope to include all cables. For example, the bounds on the value of $\tau$ on $(m, m r+1)$-cables described in Theorem 1 extend to all cables as follows.

Corollary 3 Let $K \subset S^{3}$ be a nontrivial knot. Then the following inequality holds for all $n$ relatively prime to $m$ :

$$
m \tau(K)+\frac{(m-1)(n-1)}{2} \leq \tau\left(K_{m, n}\right) \leq m \tau(K)+\frac{(m-1)(n+1)}{2} .
$$

When $K$ satisfies $\tau(K)=g(K)$, we have

$$
\tau\left(K_{m, n}\right)=m \tau(K)+\frac{(m-1)(n-1)}{2},
$$

whereas when $\tau(K)=-g(K)$, we have

$$
\tau\left(K_{m, n}\right)=m \tau(K)+\frac{(m-1)(n+1)}{2} .
$$


Observe that the results in Corollary 3 could probably also have been obtained by using the definition of $\tau$ and studying the filtered chain homotopy type of $\mathcal{F}\left(K_{m, n}\right)$ for $n$ relatively prime to $m$. However, the proof here avoids this and uses only the analysis of $\mathcal{F}\left(K_{m, m r+1}\right)$ in [3] together with Theorem 2 to obtain the result for all cables.

The second half of Corollary 3 motivates studying knots $K$ for which $\tau(K)=g(K)$. Hedden summarized many results about such knots and their $(m, m r+1)$-cables in [3]. Now combining that discussion with Corollary 3 from above, we can extend several of his results to a more general setting. Let $\mathcal{P}$ denote the class of all knots satisfying the equality $\tau(K)=g(K)$. An immediate consequence of Corollary 3 is the following.

Corollary 4 Let $K$ be a nontrivial knot in $S^{3}$, and let $n$ be relatively prime to $m$.

(1) If $K \in \mathcal{P}$, then $K_{m, n} \in \mathcal{P}$ if and only if $n>0$.

(2) If $K \notin \mathcal{P}$, then $K_{m, n} \notin \mathcal{P}$.

As discussed in [3], $\mathcal{P}$ contains several classes of knots. We mention two such classes here:

- Any knot $K$ which bounds a properly embedded complex curve, $V_{f} \subset B^{4}$, with $g\left(V_{f}\right)=g(K)$. This set of knots includes, for example, positive knots (that is, knots which admit diagrams with only positive crossings). (See Hedden [1] and Livingston [9].)

- Any knot which admits a positive lens space (or L-space) surgery. (See Ozsváth and Szabó [12].)

From this, we have the following immediate applications extending the work of [3].

Corollary 5 If $K_{m, n}$ bounds a properly embedded complex curve $V_{f} \subset B^{4}$ satisfying $g\left(V_{f}\right)=g\left(K_{m, n}\right)$, then $n>0$ and $\tau(K)=g(K)$.

Corollary 6 Suppose that $K_{m, n}$ admits a positive lens space (or $L-$-space) surgery. Then $n>0$ and $\tau(K)=g(K)$.

Corollary 7 Suppose $K \notin \mathcal{P}$. Then $K_{m, n}$ is not a positive knot for any relatively prime pair of integers $m, n$.

A final corollary concerns a more general class of knots - the class of $\mathbb{C}-$ knots. A knot $K$ is a $\mathbb{C}$-knot if $K$ bounds a properly embedded complex curve $V_{f} \subset B^{4}$. From $[1 ; 13 ; 16]$, we know that for such knots, $\tau(K)=g_{4}(K) \geq 0$. Coupling this result with Corollary 3 , we have the following corollary. 
Corollary 8 Suppose that $K_{m, n}$ is a $\mathbb{C}-$ knot. Then $n \geq-2 m \tau(K) /(m-1)-1$.

The primary significance of each of these corollaries is that they can be used as obstructions to cables having the discussed properties. Moreover, it is interesting that $\tau$ provides obstructions to such a wide array of geometric notions. For an excellent extended discussion of this, we refer the reader to [3].

This paper is organized as follows. Section 2 contains the proof of Theorem 2. Section 3 contains the proof of Corollary 3. Finally, in Section 4 we observe that the strategy for the proof of Theorem 2 extends to a broader setting in which, instead of cabling, we consider a braiding construction.

Acknowledgments I thank both Charles Livingston and Matthew Hedden for several helpful conversations.

\section{Proof of Theorem 2}

Let $r, n$ be integers relatively prime to $m$ with $n>r$. The general strategy here is to first find a cobordism between $K_{m, n} \#-K_{m, r}$ and a torus knot.

We begin with the knot $K_{m, n} \#-K_{m, r}$. Working through signs and orientations carefully, we find that

$$
K_{m, n} \#-K_{m, r}=K_{m, n} \#(-K)_{m,-r} .
$$

We will now do a series of band moves to the knot $K_{m, n} \#(-K)_{m,-r}$. A band move on any knot $K \subset S^{3}$ is accomplished as follows. Start with an embedding $b: I \times I \longrightarrow S^{3}$ such that $b(I \times I) \cap K=b(I \times\{0,1\})$ and such that $b$ respects the orientation of $K$. Define $K_{b}=K-b(I \times\{0,1\}) \cup b(\{0,1\} \times I)$. The knot (or link) $K_{b}$ is the result of doing a band move along $b$. Doing a band move to a knot simultaneously constructs a cobordism from the knot $K$ to $K_{b}$. The genus of this cobordism can be computed explicitly. For example, in the special case that the result of performing a sequence of band moves is again a knot, one can show that the genus of the cobordism is half of the number of bands added.

Now there is a sequence of $m-1$ band moves on $K_{m, n} \#(-K)_{m,-r}$ which results in the knot (or link) $(K \#-K)_{m, n-r}$. See Figure 1 for an example. Since $K \#-K$ is cobordant to the unknot, $(K \#-K)_{m, n-r}$ is cobordant to the torus link $T_{m, n-r}$. Let $k_{+}$denote the smallest positive integer such that $n-r+k_{+}$is relatively prime to $m$. (If $n-r$ is already relatively prime to $m$, then set $k_{+}=0$.) By doing $k_{+} \cdot(m-1)$ additional band moves to the torus link $T_{m, n-r}$, we obtain the torus knot $T_{m, n-r+k_{+}}$ 

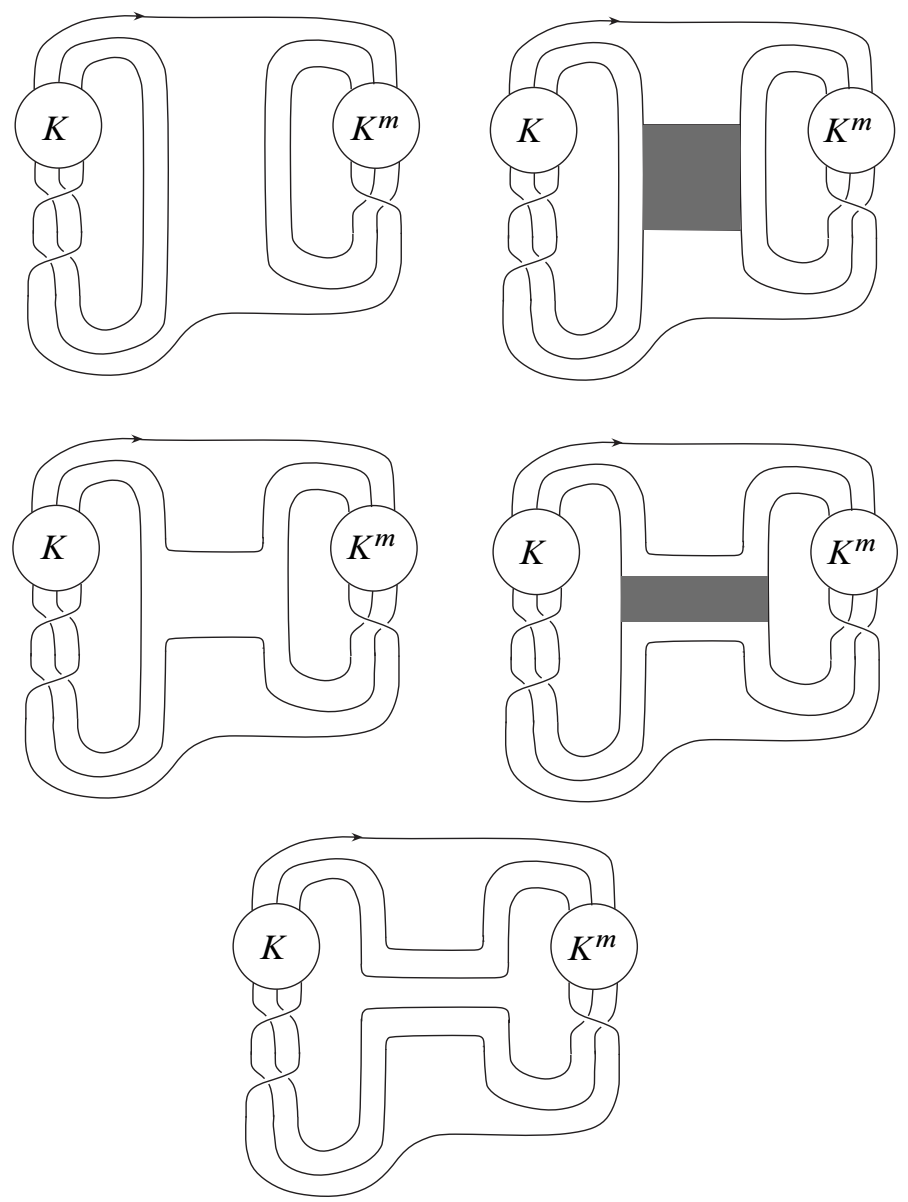

Figure 1: Beginning with the knot $K_{3,2} \#(-K)_{3,-1}$, we perform two band moves and obtain the knot $(K \#-K)_{3,1} . K^{m}$ denotes the mirror image of $K$.

(Figure 2). Altogether, the total number of band moves performed was $\left(k_{+}+1\right)(m-1)$. Therefore, the knot $K_{m, n} \#-K_{m, r}$ is genus $\left(k_{+}+1\right)(m-1) / 2$ cobordant to the torus knot $T_{m, n-r+k_{+}}$. Hence we conclude that

$$
g_{4}\left(K_{m, n} \#-K_{m, r} \#-T_{m, n-r+k_{+}}\right) \leq \frac{\left(k_{+}+1\right)(m-1)}{2} .
$$

Now since $|v(K)| \leq g_{4}(K)$, it follows that

$$
\left|v\left(K_{m, n} \#-K_{m, r} \#-T_{m, n-r+k_{+}}\right)\right| \leq \frac{\left(k_{+}+1\right)(m-1)}{2} .
$$




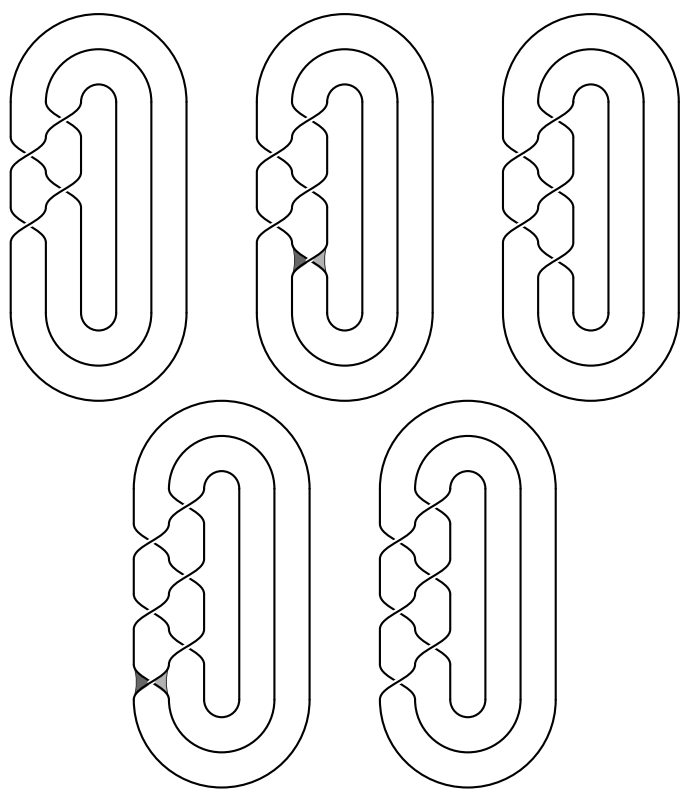

Figure 2: Beginning with the torus knot $T_{3,2}$, we perform two band moves and obtain $T_{3,3}$.

Simplifying the expression using the properties of $v$, we obtain

$$
\left|v\left(K_{m, n}\right)-v\left(K_{m, r}\right)-\frac{(m-1)\left(n-r+k_{+}-1\right)}{2}\right| \leq \frac{\left(k_{+}+1\right)(m-1)}{2} .
$$

At this point, recall the function $h(n)$ which we defined earlier. Using the definition of $h$, we can further simplify the inequality:

$$
\left|h(n)-h(r)-\frac{(m-1)\left(k_{+}-1\right)}{2}\right| \leq \frac{\left(k_{+}+1\right)(m-1)}{2} .
$$

Hence,

$$
-(m-1) \leq h(n)-h(r) \leq k_{+}(m-1) .
$$

Notice that if $k_{+}=0$, then we are done. If not, then we continue as follows.

Similar to before, let $k_{-}$denote the largest negative integer such that $n-r+k_{-}$is relatively prime to $m$. By doing $\left|k_{-}\right| \cdot(m-1)$ band moves to $T_{m, n-r}$, we can obtain the torus knot $T_{m, n-r+k_{-}}$. Proceeding through the same steps as before, we obtain

$$
\left(k_{-}-1\right)(m-1) \leq h(n)-h(r) \leq 0 .
$$


Combining (1) and (2), we have

$$
-(m-1) \leq h(n)-h(r) \leq 0
$$

for all integers $n>r$ where both $n$ and $r$ are relatively prime to $m$.

\section{Proof of Corollary 3}

Combining Theorem 1 and Theorem 2 together, we obtain an easy proof that the bounds on the value of $\tau$ on $(m, m r+1)$-cables described in Theorem 1 extend to all cables. We now restate and prove Corollary 3.

Corollary 3 Let $K \subset S^{3}$ be a nontrivial knot. Then the following inequality holds for all $n$ relatively prime to $m$ :

$$
m \tau(K)+\frac{(m-1)(n-1)}{2} \leq \tau\left(K_{m, n}\right) \leq m \tau(K)+\frac{(m-1)(n+1)}{2} .
$$

When $K$ satisfies $\tau(K)=g(K)$, we have

$$
\tau\left(K_{m, n}\right)=m \tau(K)+\frac{(m-1)(n-1)}{2},
$$

whereas when $\tau(K)=-g(K)$, we have

$$
\tau\left(K_{m, n}\right)=m \tau(K)+\frac{(m-1)(n+1)}{2} .
$$

Proof The proof of this corollary is obtained by carefully combining the equalities and inequalities found in Theorem 1 and Theorem 2. We will demonstrate a portion of the proof, leaving the rest to the reader.

Let $m$ and $n$ be two relatively prime integers with $m>0$. Let $r$ be an integer such that $n>m r+1$. Then by Theorem 2 ,

$$
h(n)-h(m r+1) \leq 0 .
$$

Using the definition of $h$ and letting $v=\tau$, we obtain

$$
\tau\left(K_{m, n}\right) \leq \tau\left(K_{m, m r+1}\right)-\frac{m-1}{2}(m r-n+1) .
$$

Using the upper bound on $\tau\left(K_{m, m r+1}\right)$ given by Theorem 1 , we have

$$
\tau\left(K_{m, n}\right) \leq m \tau(K)+\frac{(m-1)(n+1)}{2},
$$

which is one side of the desired inequality. 
To obtain the other side of the inequality, let $r^{\prime}$ be an integer such that $m r^{\prime}+1>n$. Then by Theorem 2,

$$
h\left(m r^{\prime}+1\right)-h(n) \leq 0 .
$$

We leave to the reader the task of reducing this inequality (using methods exactly similar to above) to obtain the desired second half of the inequality in the corollary.

Now let $K$ be a knot such that $\tau(K)=g(K)$. Suppose for contradiction that $\tau\left(K_{m, n}\right) \neq$ $m \tau(K)+(m-1)(n-1) / 2$. By the inequality discussed above, this implies that $\tau\left(K_{m, n}\right)>m \tau(K)+(m-1)(n-1) / 2$. Again, let $r$ be an integer such that $n>m r+1$. Then we have

$$
\begin{aligned}
h(n)-h(m r+1) & =\tau\left(K_{m, n}\right)-\frac{(m-1)}{2} n-\tau\left(K_{m, m r+1}\right)+\frac{m-1}{2}(m r+1) \\
& =\tau\left(K_{m, n}\right)-\frac{(m-1)}{2} n-m \tau(K)+\frac{(m-1)}{2} \\
& >m \tau(K)+\frac{(m-1)(n-1)}{2}-\frac{(m-1)}{2} n-m \tau(K)+\frac{(m-1)}{2} \\
& =0 .
\end{aligned}
$$

This contradicts Theorem 2. Therefore, $\tau\left(K_{m, n}\right)=m \tau(K)+(m-1)(n-1) / 2$ for all $n$ relatively prime to $m$. A similar argument settles the case when $K$ is a knot such that $\tau(K)=-g(K)$.

\section{Further analysis}

The process of cabling a knot can be reinterpreted as a special case of the following more general procedure. Let $\beta$ be an element of the braid group $B_{m}$ such that the closure of the braid $\hat{\beta}$ is a knot. There is a natural solid torus $V$ which contains the closed braid $\hat{\beta}$. Remove a neighborhood of a knot $K$ in $S^{3}$ and glue in the solid torus $V$ by a homeomorphism which maps longitude to longitude and meridian to meridian. We denote the resulting knot by $K_{\beta}$. Notice that if we take the braid $\beta \in B_{m}$ to be $\left(\sigma_{m-1} \sigma_{m-2} \cdots \sigma_{1}\right)^{n}$ (where $\sigma_{i}$ denotes the $i$-th standard generator of the braid group), then the resulting knot $K_{\beta}$ is the $(m, n)$-cable $K_{m, n}$.

For any braid $\beta \in B_{m}$, let $\beta_{r}$ denote the braid consisting of $\beta$ with $r$ full twists adjoined to the end of the braid. Specifically, $\beta_{r}=\beta\left(\sigma_{m-1} \sigma_{m-2} \cdots \sigma_{1}\right)^{m r}$. The value of $v$ on $K_{\beta_{r}}$ as a function of $r$ turns out to have controlled behavior similar to that of cabling. Define the function

$$
g(r)=v\left(K_{\beta_{r}}\right)-\frac{(m-1)}{2} m r,
$$


where $\beta \in B_{m}$ is a braid whose closure is a knot and $r$ is an integer. Then we have the following theorem about the behavior of the function $g$.

Theorem 9 The function $g(r)$ is a nonincreasing integer valued function which is bounded below. In particular,

$$
-(m-1) \leq g(r)-g(s) \leq 0
$$

for all $r>s$.

From this theorem, it follows that the function $g$ is eventually constant. This allows us to describe quite clearly a relationship among the values of $\tau$ (and $s$ ) on an entirely new set of knots. Fixing a knot $K$ and a braid $\beta \in B_{m}$ such that $\widehat{\beta}$ is a knot, Theorem 9 implies that for all large $r$,

$$
v\left(K_{\beta_{r+1}}\right)=v\left(K_{\beta_{r}}\right)+\frac{m(m-1)}{2} .
$$

where $v$ can be taken to be either $\tau$ or $s$. Note that if we take $K$ in the above construction to be the unknot, then the theorem relates the values of $v$ on knots with braid representatives which differ by full twists.

We turn now to the proof of Theorem 9.

Proof As with the proof of Theorem 2, the first goal here is to find a cobordism between $K_{\beta_{r}} \#-K_{\beta_{s}}$ and a torus knot. Notice that $-K_{\beta_{s}}=(-K)_{\left(\beta^{-1}\right)_{-s}}$. Therefore,

$$
K_{\beta_{r}} \#-K_{\beta_{s}}=K_{\beta_{r}} \#(-K)_{\left(\beta^{-1}\right)_{-s}} .
$$

By doing $m-1$ band moves to the latter knot, we obtain the knot $\left.(K \#-K)_{\left(\beta \beta^{-1}\right.}\right)_{r-s}$. Since $K \#-K$ is cobordant to the unknot and $\beta \beta^{-1}$ is the trivial $m$-strand braid, this new knot is cobordant to the torus link $T_{m, m(r-s)}$. Again, by doing $(m-1)$ band moves to the torus link $T_{m, m(r-s)}$, we obtain the torus knot $T_{m, m(r-s)+1}$. A total of $2(m-1)$ band moves have been performed. Therefore, the knot $K_{\beta_{r}} \#-K_{\beta_{s}}$ is genus $(m-1)$ cobordant to the torus knot $T_{m, m(r-s)+1}$. Hence

$$
g_{4}\left(K_{\beta_{r}} \#-K_{\beta_{s}} \#-T_{m, m(r-s)+1}\right) \leq m-1 .
$$

Now since $|v(K)| \leq g_{4}(K)$, it follows that

$$
\left|v\left(K_{\beta_{r}} \#-K_{\beta_{s}} \#-T_{m, m(r-s)+1}\right)\right| \leq m-1,
$$

which simplifies to

$$
\left|v\left(K_{\beta_{r}}\right)-v\left(K_{\beta_{s}}\right)-\frac{(m-1) m(r-s)}{2}\right| \leq m-1 .
$$


We now recall the function $g(r)$ which we defined earlier. Using the definition of $g$, we can further simplify the inequality and obtain

$$
-(m-1) \leq g(r)-g(s) \leq m-1 .
$$

This gives us only half of the desired inequality. To obtain the remaining half, go back to the torus link $T_{m, m(r-s)}$ which we obtained from $K_{\beta_{r}} \#-K_{\beta_{s}}$ by a cobordism which added $m-1$ bands. Instead of adding $m-1$ additional bands to obtain the torus knot $T_{m, m(r-s)+1}$, add $m-1$ bands to obtain the torus knot $T_{m, m(r-s)-1}$. Proceeding through the same steps as before, we obtain

$$
-2(m-1) \leq g(r)-g(s) \leq 0 .
$$

Combining (3) and (4), we have

$$
-(m-1) \leq g(r)-g(s) \leq 0
$$

for all integers $r>s$, as desired.

\section{References}

[1] M Hedden, Notions of positivity and the Ozsváth-Szabó concordance invariant arXiv:math/0509499

[2] M Hedden, On knot Floer homology and cabling, Algebr. Geom. Topol. 5 (2005) 1197-1222 MR2171808

[3] M Hedden, On knot Floer homology and cabling. II, Int. Math. Res. Not. (2009) 2248-2274 MR2511910

[4] M Hedden, P Ording, The Ozsváth-Szabó and Rasmussen concordance invariants are not equal, Amer. J. Math. 130 (2008) 441-453 MR2405163

[5] C Kearton, The Milnor signatures of compound knots, Proc. Amer. Math. Soc. 76 (1979) 157-160 MR534409

[6] W B R Lickorish, An introduction to knot theory, Graduate Texts in Math. 175, Springer, New York (1997) MR1472978

[7] R A Litherland, Signatures of iterated torus knots, from: "Topology of lowdimensional manifolds (Proc. Second Sussex Conf., Chelwood Gate, 1977)", (R A Fenn, editor), Lecture Notes in Math. 722, Springer, Berlin (1979) 71-84 MR547456

[8] R A Litherland, Cobordism of satellite knots, from: "Four-manifold theory (Durham, N.H., 1982)", (C Gordon, R Kirby, editors), Contemp. Math. 35, Amer. Math. Soc. (1984) 327-362 MR780587

[9] C Livingston, Computations of the Ozsvath-Szabó knot concordance invariant, Geom. Topol. 8 (2004) 735-742 MR2057779 
[10] P Ozsváth, Z Szabó, Knot Floer homology and the four-ball genus, Geom. Topol. 7 (2003) 615-639 MR2026543

[11] P Ozsváth, Z Szabó, Holomorphic disks and knot invariants, Adv. Math. 186 (2004) 58-116 MR2065507

[12] P Ozsváth, Z Szabó, On knot Floer homology and lens space surgeries, Topology 44 (2005) 1281-1300 MR2168576

[13] O Plamenevskaya, Bounds for the Thurston-Bennequin number from Floer homology, Algebr. Geom. Topol. 4 (2004) 399-406 MR2077671

[14] J A Rasmussen, Khovanov homology and the slice genus arXiv:math/0402131

[15] J A Rasmussen, Floer homology and knot complements, $\mathrm{PhD}$ thesis, Harvard University (2003)

[16] L Rudolph, Algebraic functions and closed braids, Topology 22 (1983) 191-202 MR683760

[17] Y Shinohara, On the signature of knots and links, Trans. Amer. Math. Soc. 156 (1971) 273-285 MR0275415

Department of Mathematics, University of San Francisco

San Francisco, California, 94117

cvancott@usfca.edu

Received: 28 December 2009 